\title{
PROTECTION AND RESTORATION STRATEGIES IN WDM MESH NETWORIKS
}

\author{
Giulia Conte ${ }^{1}$, Marco Listanti ${ }^{2}$, Marina Settembre ${ }^{3}$, Roberto Sabella ${ }^{3}$ \\ ${ }^{1}$ Coritel, Rome, Italy \\ 2 Infocom Dpt-University of Rome "La Sapienza", Rome, Italy \\ ${ }^{3}$ Ericsson Lab, Rome, Italy
}

\begin{abstract}
This paper analyzes strategies for protection and restoration in a WDM network supporting two main classes of services. We refer to a scenario in which the optical network interacts with Multi-Protocol Label Switching (MPLS) based networks. We analyze routing, wavelength assignment, and protection/restoration issue. First the analysis is done at a pure optical level, handling the traffic with wavelength granularity. Then the option of a routing coordinated between the MPLS and optical layers is taken into account. In this case, there is the possibility to route the traffic on a LSP basis, providing a more efficient use of optical resources and restoration capabilities at the expense of an increase of electrical processing.
\end{abstract}

Key words: Protection, restoration, WDM, multi-layer routing

\section{INTRODUCTION}

WDM-based mesh network infrastructures are likely to provide high capacity and cost effective transport network. The advantage of high speed and high network connectivity should be accompanied by adequate network survivability features. In fact, in case of a link failure a tremendous amount of information can be lost, affecting a huge number of customers' requests. In this scenario network survivability becomes an important issue. Moreover thanks to the feasibility of new emerging optical technology and devices (as Optical Cross Connects, OXC, and Optical Add and Drop Multiplexer, OADM) the optical layer is not only able to provide capacity but also to implement some network functionalities, leading to a migration from pointto-point WDM systems towards wavelength routed networks. 
The main network functionalities that can be performed at the optical layer are: to provide connections with QoS to the upper layers or client networks, to protect and/or restore the traffic in case of a failure, monitoring and detection of failures. The scope of this paper is mainly focused on the first two issues, that strictly relate to the routing and wavelength assignment problems in WDM networks, supporting traffic demands with different quality of service (QoS) and implementation of efficient protection and restoration strategies.

Traffic can be classified in several classes of services, according to different requirements in terms of quality of service (QoS). For sake of simplicity, here only two classes of services are considered: i) High Priority (HP) traffic, demanding for protection against failures, and ii) Low Priority (LP) traffic, which denotes typical IP services which are treated in a best effort way, with the possibility of preemption.

Routing functionality can be exploited separately or coordinately with the upper protocol layers, depending on the architectural reference scenario. In the literature basically two different architectural models have been proposed to support the evolution of IP over Optical Networks: the overlay model and the peer model [1]. In the overlay model the optical network interacts with networks based on MPLS, with a separate control plane: that is the optical network manages its resources to better serve the traffic coming from the MPLS networks. At the optical network ingress, MPLS edge routers perform the aggregation of different Label Switched Paths (LSPs) onto a same wavelength, and the opposite operation at the optical network egress. In this case the traffic is segregated on a wavelength basis. In the Peer model the MPLS and Optical layers are controlled and managed in an integrated and homogeneous way and a coordinated routing between the two layers is possible leading to a traffic segregation both on a LSP and wavelength basis. More efficient resource utilization is possible at the expense of a more sophisticated routing and signaling.

In this paper we first investigate a scenario in which pure optical routing is considered and, in this context, different protection and restoration strategies have been compared in case of a single link failure. Secondly, we consider a scenario in which a coordination, between the WDM and MPLS layers, is possible.

This paper is organized as follows. Section 2 introduces a sort of road map and terminology related to protection/ restoration aspects in WDM networks in order to better understand where the choice presented in this paper is located within a wider scenario. The adopted protection schemes are also briefly described. In section 3 the main hypothesis on which our analysis is based are defined and the analysis procedure is explained both in 
the case of single-layer and multi-layer routing. Results are shown in section 4 and finally some conclusions are derived in section 5 .

\section{CLASSIFICATION OF THE PROTECTION/RESTORATION SCHEMES}

Many fault recovery mechanisms have been proposed for generic meshed networks in the literature. They can be classified by the route computation, by the execution mechanism (centralized or distributed), by the layer where they take place (WDM, MPLS, IP...), by the type of protection (link-based or path-based), and by the computation timing (precomputed or real time). Referring to [3] we can schematically consider two main categories: protection and restoration techniques. The former allocates and reserves the back-up resources in advance, providing a fast recovering on preplanned paths, at the expense of an inefficient use of resources. The latter makes use of real time availability of resources, it means that protection resources are not pre-allocated, but the cost to be paid is in terms of recovery speed. It is noteworthy that the two techniques can coexist in the same network.

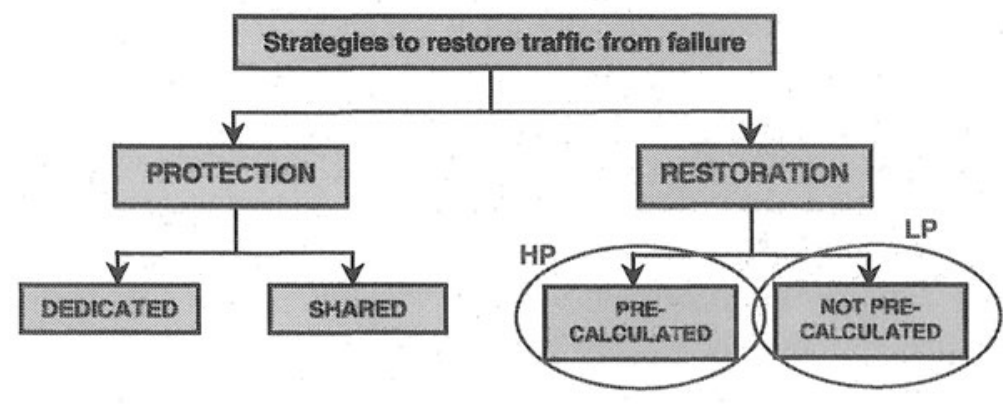

Figure 1. Protection and restoration schemes classification.

The strategies considered in this paper are highlighted with circles.

Moreover the protection techniques can be distinguished in two cases depending on the level of resources reservation: dedicated or shared schemes. In the dedicated case the protection paths cannot share the resources among them. (They are typically referred in the literature as $1+1$ or 1:1 scheme, depending if the protection path is occupied by the same traffic as the working oath or by best effort traffic). In the shared schemes several disjoint working paths can have the same protection paths (1:n or m:n [3]).

In restoration tecniques a protection path for a connection may be selected or not a priori, but, in both cases, its establishment occurs only after a failure in the working path [4]. Another classification can be done 
depending if the recovery mechanism applies to the whole path between the source and destination node or only to the link affected by the failure. In the following text we will refer to these mechanism as "end to end" and "link" schemes [5].

In fig. 1 a schematic classification is sketched and the choice adopted in this paper is highlighted. We consider pre-calculated restoration techniques for HP traffic, and a simple restoration strategy for the LP traffic. In our work, the protection strategies for HP traffic are applied with wavelength granularity, while the restoration scheme for the LP traffic will be applied both at a wavelength level and at a LSP level.

The calculation of the protection paths for HP traffic can be done in several ways. This paper analyses three of these pre-calculated restoration strategies: two end-to-end (or path) schemes (Disjoint Path and Single Link Basis) and one link protection scheme (Link Repair).
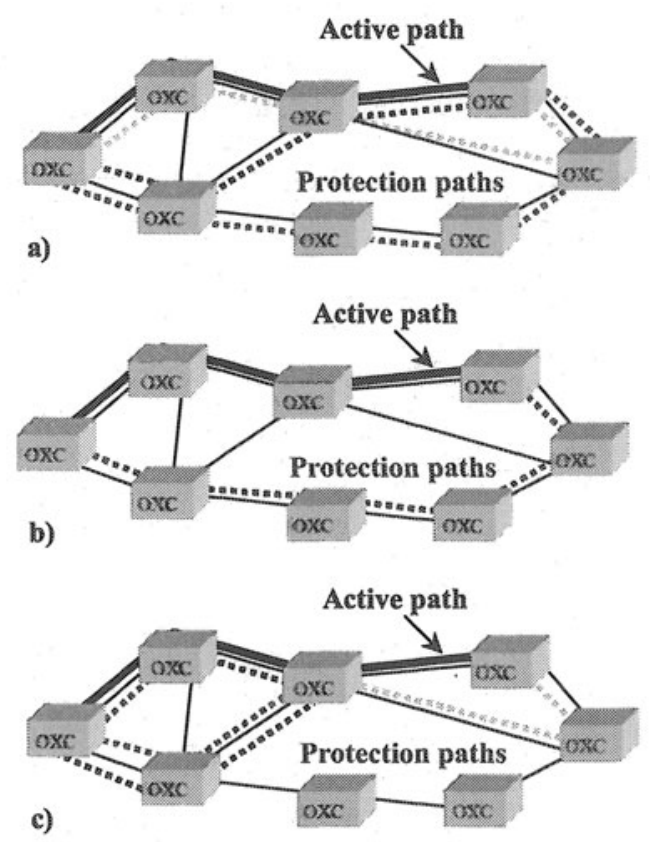

Figure 2. Optical protection strategies: a) Single Link Basis; b) Disjoint Path; c) Local Repair

In the Single Link Basis (SLB) scheme each path to be protected, consisting of a sequence of links, is associated to as many protection paths as the number of links that form the path itself. Any of these protection paths is computed in order to exclude one particular link of the active path, so that in case of failure of a link belonging to the working path, it does exist a protection path excluding that link. In this way, the protection paths can have 
one or more nodes and links in common (Fig.2a). It has to be noticed that this scheme needs a signaling system able to detect and notify which link has failed. The computational complexity is rather high.

In the Disjoint Path (DP) scheme a completely protection disjoint path is associated to the working path (Fig. $2 \mathrm{~b}$ ). The protection path is obtained by removing all the links crossed by the working path and calculating an alternative path in the new topology. The DP scheme offers a greater resource redundancy respect to the SLB scheme to protect the same number of paths, but it is not necessary to have a signaling system that notify exactly which link has failed.

The Link Repair (LR) scheme protects the links, and not the whole path, in the network. This means that a generic link has as many protection paths (that go around that link) as the number of working paths that cross the link to be protected (Fig. 2c). Depending on the network topology some of these protection paths may be identical. The LR protection scheme involves a greater resource redundancy respect to the SLB and DP schemes to protect the same number of paths. Due to the fact that the recovery is triggered from a network element near to the failure (the originating or terminating nodes of the link), we expect that the recovery time is shorter than the recovery time necessary for the end-to-end strategies.

\section{ANALYSIS PROCEDURE}

Our analysis can be subdivided in two phases. In a first step, referring to an overlay network scenario, we analyze routing, wavelength assignment and protection/restoration issue at a purely optical level, handling with a wavelength granularity. The proposed strategy consists in a network dimensioning to support both the classes of services in normal working operations and to guarantee protection just for the HP traffic in case of a failure. In the framework of this strategy, two path-based protection schemes, (Disjoint Path and Single Link Basis), and one link-based protection scheme, (Link Repair), have been compared in terms of the system scale and the percentage of LP traffic, that can be served by the optical network after a failure, including or not wavelength conversion capability at each optical node. Fixing a maximum tolareble value of LP traffic lost after a link failure, the network is dimensioned and the different restoration schemes have been compared in terms of the sytem scale.

In a second step, referring to a peer network scenario, we consider the advantage of a routing coordinated between the MPLS and optical layer routing when necessary the traffic also on a LSP basis in order to efficiently 
exploit network resources and increase restoration capabilities, even if the cost of electronic processing have to be properly taken into account.

\subsection{All-optical protection/restoration}

In this section, the procedure followed in the first phase of our analysis is described. The optical network dimensioning aims at, in normal working condition, serving both the HP and LP traffic and, in case of a single link failure, to guarantee the protection to $\mathrm{HP}$ paths and at restoring the largest amount of LP pre-empted paths at the optical level.

The logical steps adopted in the procedure are shown in Fig. 3

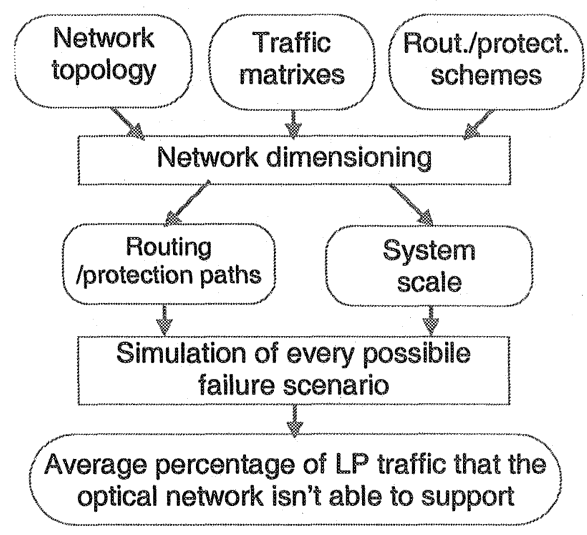

Figure 3. Analysis procedure in a network planning

The inputs of the network planning are: a) the network topology, b) two traffic matrixes relating to HP and LP traffics, respectively, c) the adopted routing/protection scheme.

The optical network topology is composed of $S$ optical cross-connects (OXCs), interconnected in a general mesh network configuration by bidirectional WDM optical links. Each link consists of multiple fibers where a single fiber can support $M$ wavelengths. The number $M$ is limited by device technology, and transmission impairments. Each fiber needs a dedicated port in an OXC. Two types of ports can be recognized in each OXC (Fig. 4): the inter-office ports and the intra-office ports. The formers support fibers incoming to or outgoing from adjacent OXCs. The latters connect the OXC to the MPLS routers in the same node; they accommodate the adding/dropping paths terminated at the node.

The traffic loading the network (traffic pattern) is represented by two path requested matrices $[H]$ and $[\mathrm{L}]$ referring to the $\mathrm{HP}$ traffic and to the $\mathrm{LP}$ 
traffic, respectively. The traffic matrices elements, $h_{i j}$ or $l_{i j}$, indicate the number of wavelength paths to be set up between the nodes $i$ and $j$.

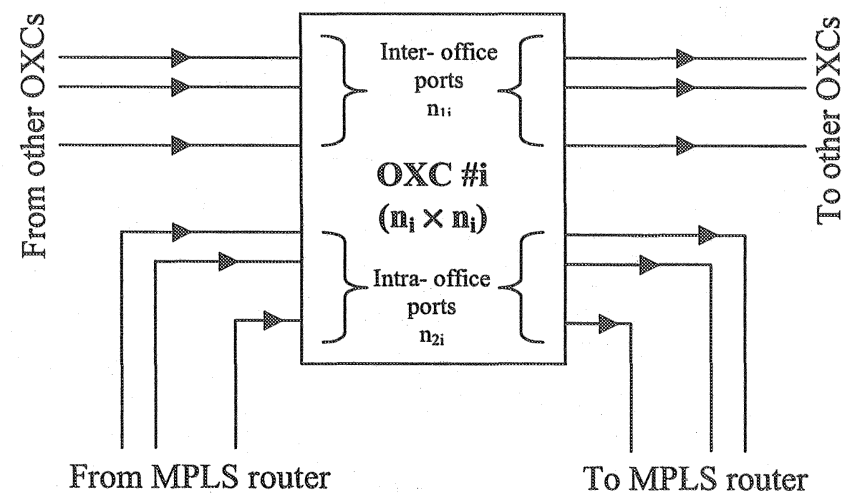

Figure 4. Generic architecture of an optical cross-connect.

The summation of all the elements of $[\mathrm{H}]$ and $[\mathrm{L}]$ is named traffic volume and corresponds to the total number of paths to be set up within the network. Each high priority path must be protected: in addition to network resources for normal working conditions, further (redundant) resources must be pre-allocated for re-routing when a failure occurs. For low priority traffic only the resources necessary to route the traffic in normal working conditions are allocated. The LP traffic can share the resources with HP protection paths, allowing an efficient use of back up capacity. In case of a failure the HP traffic can preempt the LP paths.

Two routing schemes have been considered. The first one is the: wavelength path (WP) and virtual wavelength path (VWP). WP routing scheme refers to a network where no wavelength conversion capability is assumed at the nodes and one wavelength is assigned to each path, from the source node to the destination node. As a result, both routing and wavelength assignment problem should be solved. In the VWP routing scheme the wavelengths are assigned link-by-link to each path and no wavelength assignment problem has to be solved.

The object function for routing/protection algorithms, during a networkplanning phase, can be the minimization of the number of fibers [6] [7], that means, minimizing the average number of OXC ports. Practically, the object function is given by the minimization of the system scale $(s)$, expressed by:

$$
s=\frac{1}{S} \sum_{i=1}^{S} n_{i}=\frac{1}{S} \sum_{i=1}^{S} n_{1 i}+\frac{1}{S} \sum_{i=1}^{S} n_{2 i}=N_{1}+N_{2}
$$


where $n_{1 i}$ and $n_{2 i}$ are the inter and intra office ports of the $i$-th OXCs.

The outputs of the dimensioning phase are the number of fiber for each link in the network and the routes for the active and protection paths.

A comparison among the different routing/protection strategies is performed choosing two performance parameters: the system scale and the average percentage, $P$, of torn down LP traffic in case of failure. The parameter $P$ can be evaluated from the following procedure. After the dimensioning of the network, a link failure is simulated and the HP traffic is restored along the protection paths. After all the HP traffic is restored, if there are some available resources in the optical network, new optical paths are created among the network to restore as many preempt-able traffic as possible. The preempted traffic that, however, cannot be re-routed is computed. This procedure is repeated for all the optical links in the network. The average percentage of LP traffic not served from the optical network after a link failure is so evaluated.

As expected, the protection schemes that require a greater system scale to protect the HP traffic, leads to a smaller value of lost LP traffic. It can be observed (see in the next paragraph) that the percentage of LP traffic, that the network supports in case of failure, does not increase linearly with the system scale. For that reason, it could be meaningful to compare the different strategies fixing a maximum tolerable value of lost LP traffic, $P_{\max }$, and by recalculating the system scale to accomplish this. Starting from the end of the previous procedure, if the evaluated percentage exceeds $P_{\max }$, a network re-dimensioning is achieved. After the re-dimensioning, the system scale, that allows the network to have an average percentage of lost LP traffic lower than the fixed percentage, can be evaluated. With this evaluation, we want to show the effect (in terms of system scale) of the different protection strategies for mission critical services, in a network where a minimal quantity of best effort services must be guaranteed.

\subsection{Multi-layer routing}

In the second phase of our analysis we evaluate the advantage of using a routing coordinated between MPLS and optical layer, as i.e. in the Peer model. In the following this kind of routing is refereed as multi-layer routing. In this case it is possible to route a LSP by means of an opportune sequence of optical paths using the residual bandwidth of the already established lightpaths, without setting up a new one. In order to perform multi-layer routing electronic processing should be done not only at the edge devices (as in the case of all optical routing above described), but also at intermediate nodes. As a result, the LSPs may traverse a multi-hop path, constituted by different lightpaths. Configuring the lightpaths optimally, 
without separating the network-layers, improves the quality results, but the complexity of the problem grows [8]. The multi-layer routing can be employed in many traffic-engineering (TE) problems (e.g. dynamic routing, provisioning, failure restoration...).

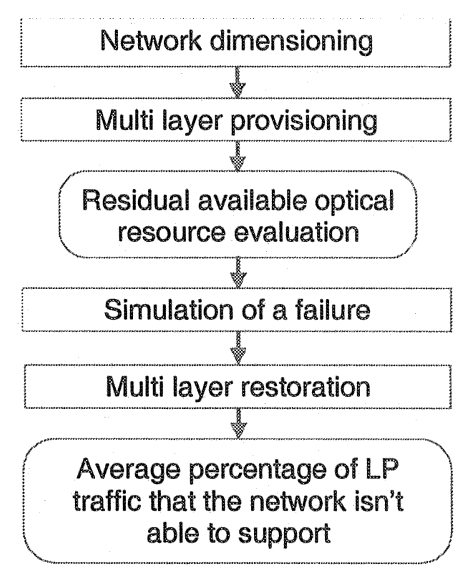

Figure 5. Analysis procedure

This section analyzes the application of multi-layer routing in semipermanent provisioning and in failure restoration problems. In semipermanent provisioning, the resources are assigned and fixed and the aim is to provide route assignment at minimum cost for a semi permanent traffic matrix. In fact, the traffic demands adopted as inputs of the provisioning procedure are expectations of the forthcoming traffic, intrinsically prone to fluctuations as well as to estimation errors. Intuitively, for a given set of traffic demands, the fewer amounts of resources a configuration solution uses, the more resources are available for allocating new routes in response to critical situation of the network with no re-arrangement of established routes. In Fig. 5, a diagram summarizes the steps of the analysis procedure. First, the network is dimensioned using the same procedure as in the previous section. Using the same traffic pattern of the dimensioning phase, the semi-permanent provisioning is achieved by using the multi-layer routing concept. The objective of the algorithm used for the multi-layer routing is to found a configuration that consumes fewer amount of network resources. The advantage, in terms of optical resources saving, is then evaluated. After a simulation of a link failure, the optical network tries to optically restore the LP traffic, exploiting also the optical resources available after the multi-layer provisioning. The multi-layer concept can also be used to further improve the quantity of restored traffic. In fact, when the optical layer is no longer able to restore traffic, a re-routing mechanism at MPLS layer can be 
employed. The lambdas, which cannot be restored, are de-multiplexed. The MPLS layer tries to re-route the single LSP using the residual bandwidth of the already established lightpaths. The restored traffic can across one or more lightpaths among its path. In our analysis, the number of optical hops used in the restoration is the same number used in the provisioning. Practically, a "bottom up" escalation strategy is adopted [10]: first, the re-routing acts at the lower layer, then, if it is necessary, the upper layer is involved in the re-routing operations.

\section{RESULTS}

In this section, the results relative to all-optical routing (section 4.1) and the results relative to multi-layer routing (section 4.2) are reported. Since the performance parameters of our analysis depend also on the distribution between HP and LP traffic loads throughout the network, we introduce the parameter $\mathrm{F}$ as the percentage of HP traffic with respect to the total traffic volume. Moreover, to avoid that the analysis also depends on a specific traffic pattern, for each value of $\mathrm{F}$, the performance parameters have been averaged among ten different traffic patterns. We report the results arising from a network topology that consists of 21 nodes and 36 links. Two different number of wavelength per fiber $(M)$ are considered: 4 and 32 .

\subsection{All-optical protection/restoration: some results}

A comparison of the different protection strategies for mission critical services, in a network where a minimal quantity of best effort services must be guaranteed is done in this section. In fig. 6 the percentage of LP paths that are torn down after a link failure and the system scale versus the parameter $F$ are reported for all the considered cases of protection scheme (DP, SLB, and LR) and wavelength routing schemes (i.e. VWP and WP)

The scheme that requires a greater system scale, leads to a smaller value of lost traffic. When $F$ is small enough (i.e. $F<0.5$ in the case of $M=4$, and $F$ $<0.3$ in the case of $M=32$ ) the network dimensioning is mainly affected by the active paths of both LP and HP traffics. Therefore, the system scale tends to be independent of $\mathrm{F}$. As $\mathrm{F}$ increases, more $\mathrm{HP}$ paths exercise their preemption rights, leading to an increment of lost LP traffic. Consequently, the percentage of lost LP traffic after a failure tends to increase with F: in fact, the increase of HP paths leads to an increase of the preemptions in case of failure. Conversely for $\mathrm{F}>0.5$ in the case of $\mathrm{M}=4$, and for $\mathrm{F}>0.3$ in the case of $M=32$, the network redundancy is such that the percentage of lost LP paths tends to decrease with $\mathrm{F}$. 
a) Average percentage of LP traffic torn down after a link failure
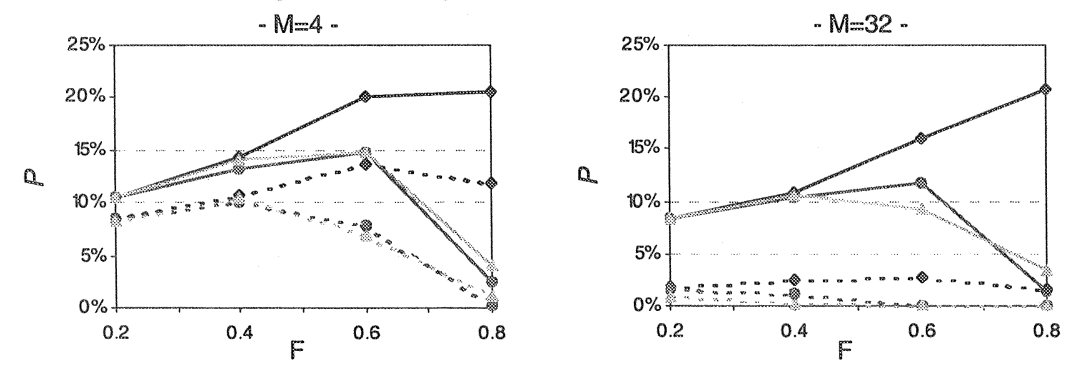

b) System scale
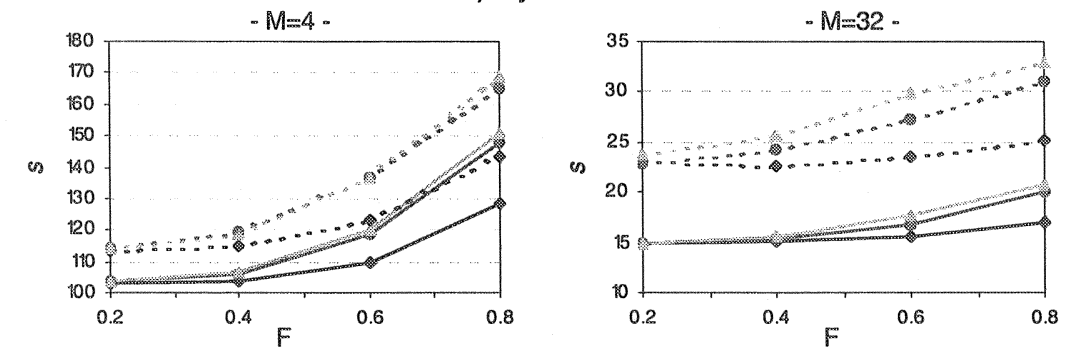

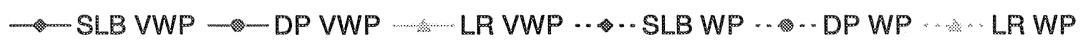

Figure 6. a) Average percentage of LP traffic torn down in case of link failure vs the percentage of HP traffic with respect to the total traffic; b) system scale vs the percentage of $\mathrm{HP}$ traffic with respect to the total traffic. $\mathrm{M}$ is the number of wavelength multiple in a fiber.

\section{System scale after re-dimensioning}
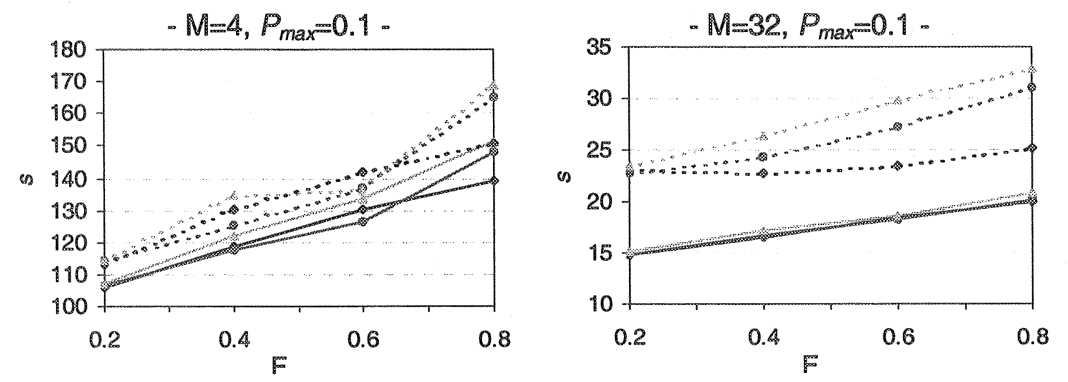

$\rightarrow$ SLB VWP $\rightarrow$ DP VWP $\cdots$ LR VWP …

Figure 7. System scale needed to obtain an average percentage of LP traffic torn down in case of link failure, less than Pmax $=10 \%$ vs the percentage of HP traffic with respect to the total traffic volume. $M$ is the number of wavelength multiplexed in a fiber. 
The SLB scheme presents the minimum values of system scale with respect to the other protection schemes, in both the WP and VWP cases. As expected, wavelength conversion leads to a minimum resource redundancy, in terms of system scale. This confirms the results reported in [6] and [7].

In order to easily compare the strategies it can be helpful to fix the maximum tolerable percentage, $P_{\max }$, of torn down LP traffic and recalculating the system scale to accomplish this.

Fig. 7 depicts the system scale, after the re-dimensioning procedure, for $P_{\max }$ equal to $10 \%$. The strategies, which require the smallest rescaling, are the SLBVWP and DPVW. They show very similar behavior, but DP strategy is preferred since it does not require an exact localization of the failure. In the second phase of the analysis only DP protection scheme is considered.

\subsection{Multi-layer routing: some results}

The advantage of using a multi-layer routing with respect to an all-optical routing in the provisioning phase is evaluated in terms of wavelength utilization coefficient expressed as follows:

$$
C=\frac{1}{N L} \cdot \sum_{i=1}^{N L} \frac{\lambda_{i}}{\lambda_{\text {TOT }_{i}}}
$$

where NL is the total number of optical links in the network; $\lambda_{i}$ the number of utilized wavelengths on the $i$-th link; $\lambda_{T O T i}$ the total number of allocated wavelengths on the $i$-th link.

Fig. 8 shows the wavelengths utilization coefficient versus the parameter F. The different curves refer to a different number of lightpaths (hops) that an LSP is allowed to cross. It can be quantitatively estimated the advantage of using a multi-layer provisioning with respect to the all-optical case (single-layer provisioning): for $\mathrm{F}=0.5$ the resources saving is more than $10 \%$ even when only two hops are allowed.

After a link failure, the increased available optical resources can be employed to optically restore a greater amount of the LP traffic. Fig. 9 shows the average percentage of restored LP traffic after the optical restoration, $G$, versus the parameter $\mathrm{F}$. For $\mathrm{F}=0.5$, in the WP case, $\mathrm{G}$ is more than $10 \%$ when the LSPS are allowed to cross at most one lightpath; while, in the WP, $\mathrm{G}$ is about $30 \%$.

Adopting the bottom-up escalation strategy mentioned in section 3.2, if, after the optical restoration, not all the LP traffic, involved in the failure, has been optically restored, an electrical restoration is applied. For each not already 
restored LSP, a re-route on the residual bandwidth of the already established lightpaths, is tempted.

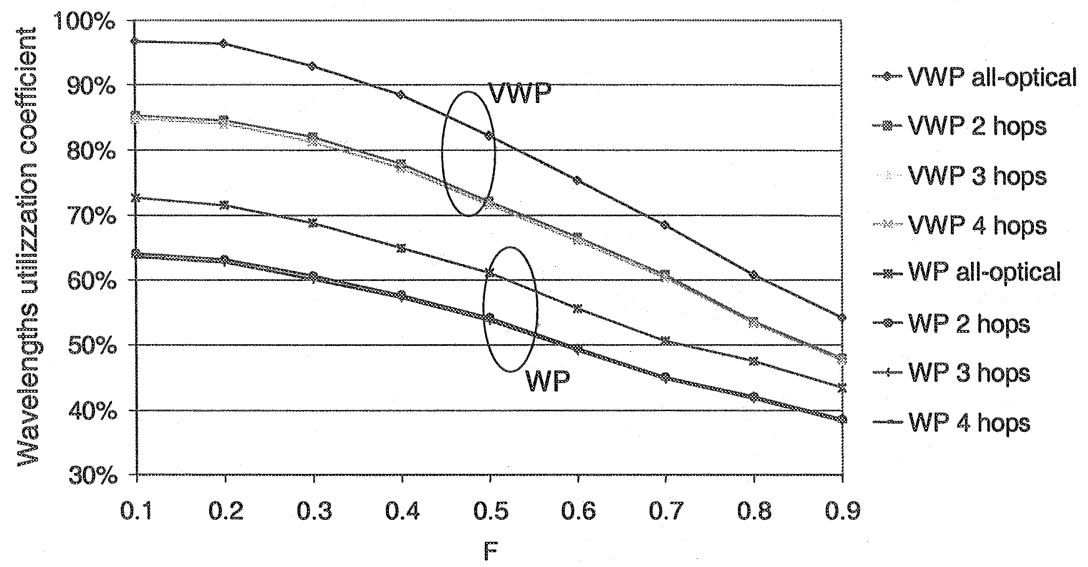

Figure 8. Wavelengths utilization coefficient vs the percentage of HP traffic with respect to the total traffic volume.

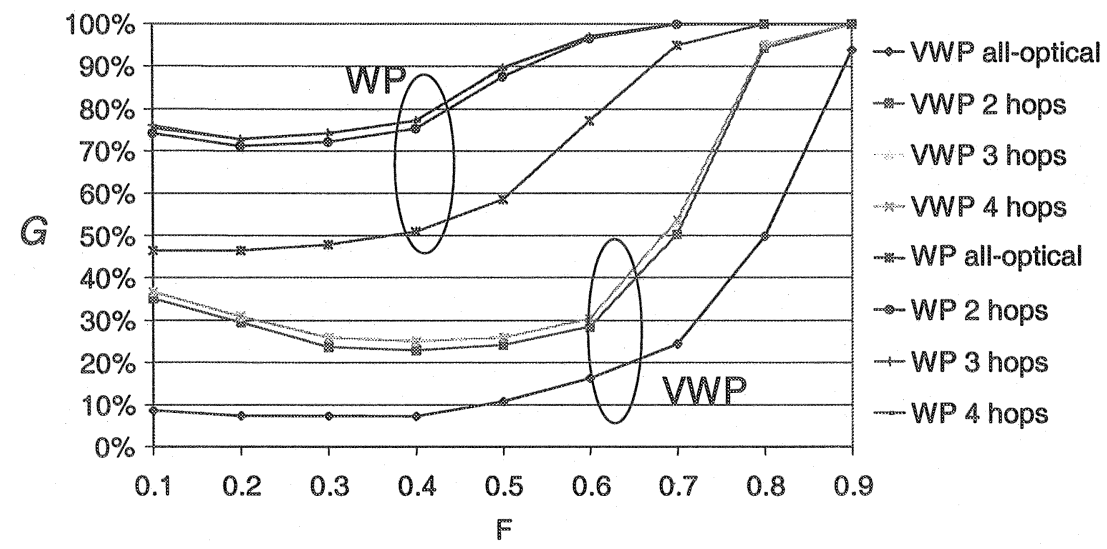

Figure 9. Average percentage of restored LP traffic after the optical restoration vs the percentage of HP traffic with respect to the total traffic volume.

Fig. 10 shows the average percentage of lost LP traffic after a failure. The curves relative to the all-optical approach (see 4.1) are also reported for comparison. It is important to notice that an all-optical provisioning with an all-optical restoration leads to the worst performance, both in presence and in absence of wavelength conversion at the optical nodes. 
Let us analyze the graphic for $\mathrm{F}=0.5$. An all-optical provisioning ( 1 hop) with a bottom-up restoration strategy can guarantee a gain of about $20 \%$ in presence of wavelength conversion, or $10 \%$ in absence of wavelength conversion, with respect to the all-optical case. The possibility to employ the multi-layer principle (more than one hop), both in the provisioning phase and in the restoration phase, leads to a gain of about $30 \%$ in presence of wavelength conversion, or $20 \%$ in absence of wavelength conversion, with respect to the all-optical case

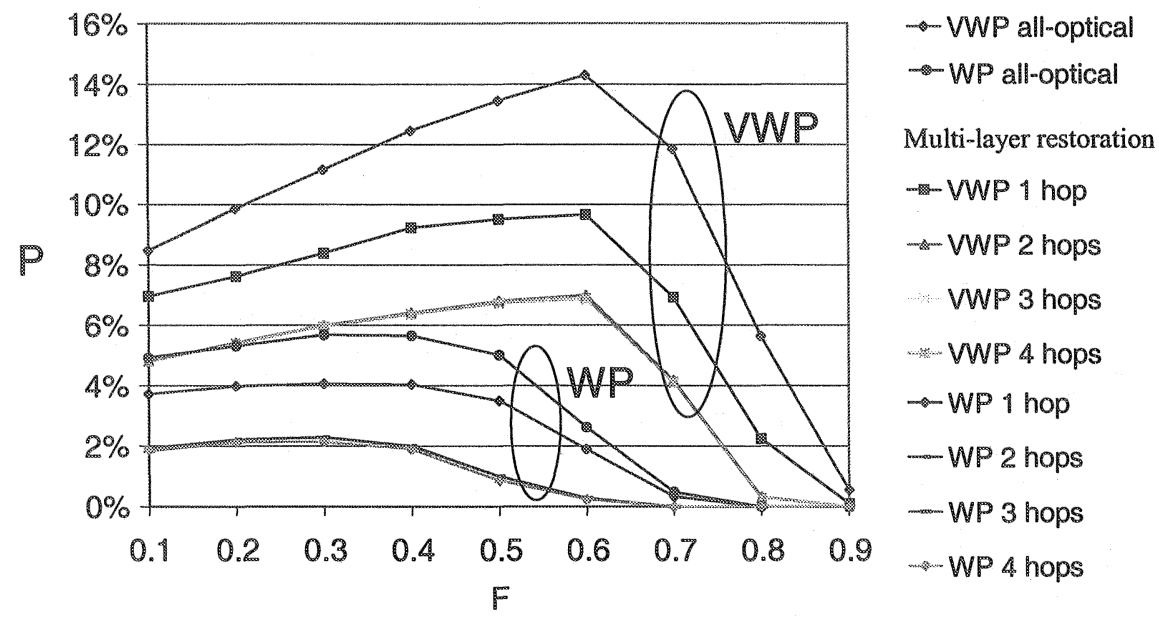

Figure 10 Average percentage of lost LP traffic vs the percentage of HP traffic with respect to the total traffic volume.

\section{CONCLUSION}

This paper analyzes protection and restoration strategies in WDM meshed networks, in presence of two class of service.

In a first step, referring to an overlay network scenario, we analyze routing, wavelength assignment and protection/restoration issue at a pure optical level, handling the traffic with wavelength granularity.

In a second step, referring to a peer network scenario, we consider the advantage of a routing coordinated among the MPLS and optical layer routing, when necessary, the traffic also on a LSP basis in order to efficiently exploit network resources.

We find out that the multi-layer provisioning allow a great saving of optical resource. These saved resources can be opportunely exploited when a failure occurs. In fact, during the optical restoration of the LP traffic, a 
greater amount of traffic can be restored if, after the provisioning phase, there are many more optical resources at disposal. Then the electrical restoration allows an increment of the restored traffic. However it has to be noticed that the system complexity and the routers load increase.

\section{ACKNOWLEDGMENT}

The authors sincerely acknowledge Gianpaolo Oriolo for the many helpful suggestions and Roberto Roccato for his support with the implementation of the algorithms.

\section{REFERENCES}

[1] B. Rajagopalan, et al., "IP over Optical Networks: Architectural Aspects", IEEE Communication Magazine, pp. 94-102, Sept. 2000.

[2] Ghani, Dixit, Wang, "On IP-over-WDM Integration", IEEE Communications Magazine, March 2000.

[3] W. S. Lai, et al., "Network Hierarchy and Multilayer Survivability", Internet Draft < draftteam-tewg-restore-hierarchy-00.txt>, work in progress, July 2001, Extirpation Date: January 2002.

[4] B. Rajagopalan, et al., "Signaling for Protection and Restoration in Optical Mesh Networks", Internet Draft <draft-bala-protecion-restoration-signalling-00.txt>, work in progress, Expires on May 2002.

[5] S. Ramamurthy and Biswanath Mukherjee, "Survivable WDM mesh networks, Part IProtection", Communications, 1999. ICC '99. IEEE International Conference on, 1999, pp. $2023-2030$, vol.3, 1999.

[6] M. Listanti and R. Sabella, "Wavelength conversion in WDM optical networks: strategies and algorithms for limiting the number of converters in the optical cross-connects", Photonic Network Communications, 2:4, pp. 335-348, 2000.

[7] N. Nagatsu, S. Okamoto, K. Sato, "Optical path cross-connect system scale evaluation using path accommodation design for restricted wavelength multiplexing", IEEE Journal of Select. Areas in Comm., vol.14, No. 5, pp. 893-902, 1996.

[8] T. Cinkler, et al., "Heuristic Algorithms for Joint Configuration of the Optical and Electrical Layer in Multi-Hop Wavelength Routing Networks", IEEE Infocom 2000.

[9] Banerjee, Mukherjee, "Wavelength-Routed Optical Network: Linear Formulation, Resource Budgeting Tradeoffs, and a Reconfiguration Study", IEEE,/ACM Trans. Networking, 8, 598-607, 2000.

[10] J.Kroculick, C. Hood, "Defining Provable-correct Escalation Policies for Multilayer Network Restoration", Global Telecomunication Conference - Globecom 19999, pp. 560564. 